\title{
Radio observations and spectrum of the SNR G127.1+0.5 and its central source $0125+628$
}

\author{
D. Leahy ${ }^{1}$ and W. Tian ${ }^{1,2}$ \\ 1 Department of Physics \& Astronomy, University of Calgary, Calgary, Alberta T2N 1N4, Canada \\ e-mail: wtian@ucalgary.ca; leahy@iras.ucalgary.ca \\ 2 National Astronomical Observatories, CAS, Beijing 100012, PR China \\ Received 7 December 2005 / Accepted 23 January 2006
}

ABSTRACT

\begin{abstract}
We present new images of the Supernova Remnant (SNR) G127.1+0.5 (R5), based on the $408 \mathrm{MHz}$ and 1420 MHz continuum emission and the HI-line emission data of the Canadian Galactic Plane Survey (CGPS). The radio spectrum of the central compact source (0125+628) is analyzed in the range $178 \mathrm{MHz}-8.7 \mathrm{GHz}$, indicating a flat spectrum with synchrotron self-absorption below $800 \mathrm{MHz}$. The SNR's flux density at $408 \mathrm{MHz}$ is $17.1 \pm 1.7 \mathrm{Jy}$ and at $1420 \mathrm{MHz}$ is $10.0 \pm 0.8 \mathrm{Jy}$, corrected for flux densities from compact sources within the SNR. The SNR's integrated flux density based spectral index $\left(S_{v} \propto v^{-\alpha}\right)$ is $0.43 \pm 0.10$. The respective T-T plot spectral index (derived from the relative size of brightness temperature variations between two frequencies, see text for details) is $0.46 \pm 0.01$. There is no evidence at $1 \sigma$ for spatial variations in spectral index within G127.1+0.5. In particular, we compared the northern shell, southern shell and central diffuse region. HI observations show structures associated with the SNR in the radial velocity range of -12 to $-16 \mathrm{~km} \mathrm{~s}^{-1}$, suggesting G127.1+0.5's distance is $1.15 \mathrm{kpc}$. The estimated Sedov age is $2-3 \times 10^{4} \mathrm{yr}$.
\end{abstract}

Key words. ISM: lines and bands - ISM: supernova remnants - Galaxy: evolution

\section{Introduction}

We report new CGPS radio observations of the bilateral symmetry shell-type SNR G127.1+0.5 (R5). G127.1+0.5 is considered as a younger remnant (18000 yr, Milne 1988) for its appearance and brightness. Because compact sources are detected near the center of the SNR, there have been many studies at radio, optical and X-ray wavelengths of the SNR and the compact sources (Pauls 1977; Pauls et al. 1982; Fürst et al. 1984; Joncas et al. 1989; Xilouris et al. 1993; Kaplan et al. 2004). But its basic physical features are still uncertain, such as its distance and age and radio spectrum. G127.1+0.5's radio spectral index in frequency range of $0.4-5 \mathrm{GHz}$ has been estimated as between 0.45 and 0.6 previously (Fürst et al. 1984; Joncas et al. 1989). The spectrum of the southern shell is slightly steeper than for the northern shell based on observations with a competitive resolution but lower sensitivity than current observations. In this paper, we present the SNR's continuum images at higher sensitivity than previously at both $408 \mathrm{MHz}$ and $1420 \mathrm{MHz}$ in order to determine its flux densities and spectrum. We search the HI-line emission for detecting possible interactions of the remnant with the surrounding gas and estimating its distance and age.

\section{Observations and analysis}

The continuum and $\mathrm{HI}$ emission data sets come from the CGPS, which is described in detail by Taylor et al. (2003).
The data sets are mainly based on observations from the Synthesis Telescope (ST) of the Dominion Radio Astrophysical Observatory (DRAO). The angular resolution of the continuum images is better than $1^{\prime} \times 1^{\prime} \operatorname{cosec}(\delta)$ (HPBW) at $1420 \mathrm{MHz}$ and $3.4^{\prime} \times 3.4^{\prime} \operatorname{cosec}(\delta)$ at $408 \mathrm{MHz}$. The synthesized beam for the HI line images is as the same as for the continuum and the radial velocity resolution is $1.32 \mathrm{~km} \mathrm{~s}^{-1}$. DRAO ST observations are not sensitive to structures larger than an angular size scale of about $3.3^{\circ}$ at $408 \mathrm{MHz}$ and $56^{\prime}$ at $1420 \mathrm{MHz}$. Thus the CGPS includes data from the $408 \mathrm{MHz}$ all-sky survey of Haslam et al. (1982), sensitive to structure greater 51', and the Effelsberg 1.4 GHz Galactic plane survey of Reich et al. $(1990,1997)$, sensitive to structure with $9.4^{\prime}$ for large scale emission (the single-dish data are freely available by http://www.mpifr-bonn.mpg.de/survey.html). The low-order spacing HI data is from the single-antenna survey of the CGPS area (Higgs \& Tapping 2000) with resolution of $36^{\prime}$. See Taylor et al. (2003) for detail of the method of combining the synthesis telescopes and single dish observations.

We analyze the continuum and $\mathrm{HI}$ images of G127.1+0.5 and determine its flux densities using the DRAO export software package. For G127.1+0.5, integrated flux density's errors are found by comparing results for several different choices of background region. We also consider a systematic error of about $5 \%$ contributed by the flux calibration uncertainties at $408 \mathrm{MHz}$ and $1420 \mathrm{MHz}$. For compact sources, the flux density's errors are taken as the formal Gaussian fit errors. 

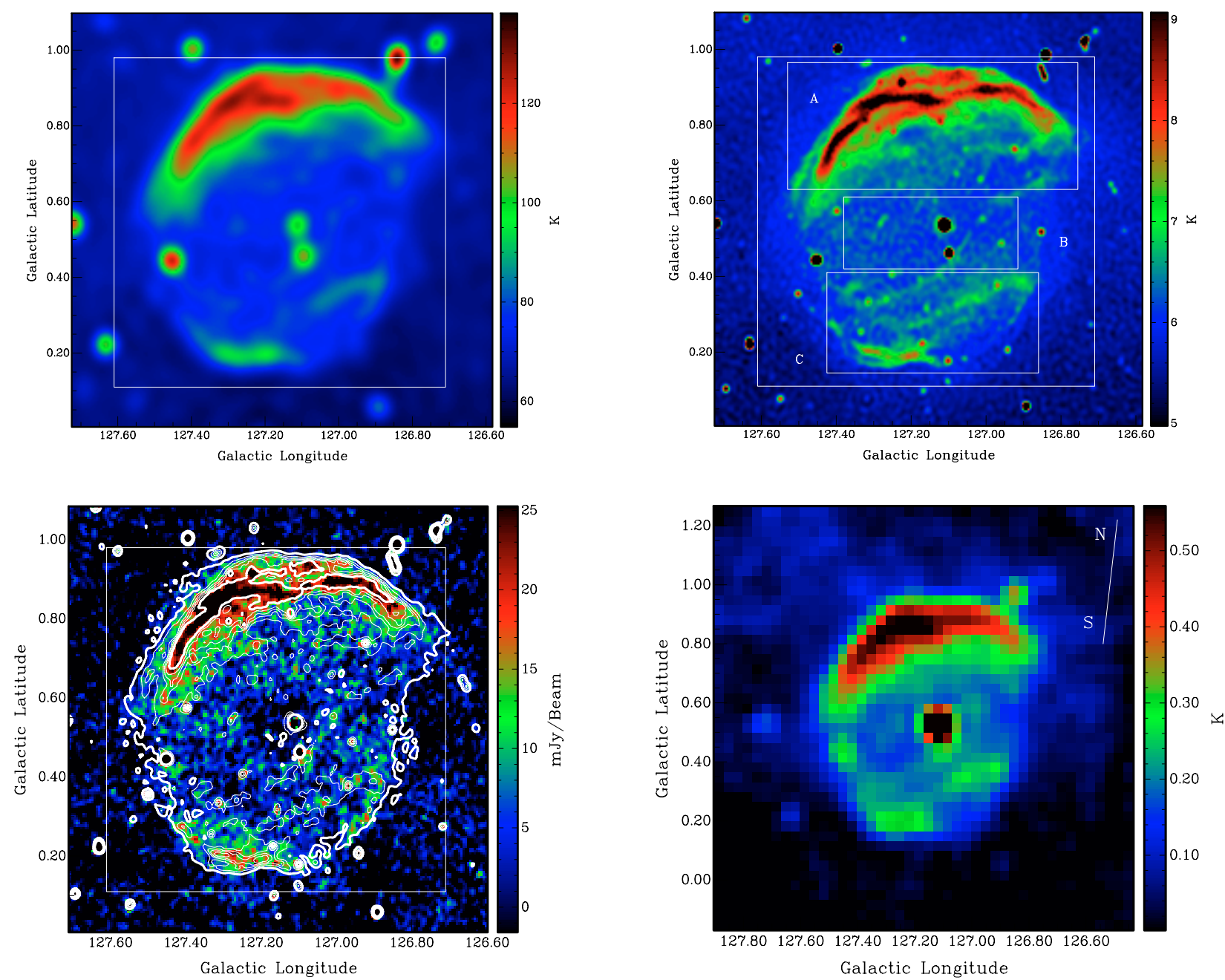

Fig. 1. The first row shows the CGPS maps at $408 \mathrm{MHz}$ (left) and $1420 \mathrm{MHz}$ (right). The second row shows at left the WENSS image at $327 \mathrm{MHz}$ (grey scale) with contours from the $1420 \mathrm{MHz}$ map (contours at 6, 6.5, 6.9, 7.2, 7.5, $8 \mathrm{~K}$ ). The second row shows at right the $2695 \mathrm{MHz}$ Effelsberg map. The box used for whole SNR T-T plots is shown in the upper left. The 3 boxes, labeled with letters and used for SNR sub-areas T-T plots, are shown in the upper right. The direction of North $(\mathrm{N})$ and South $(\mathrm{S})$ is marked on the lower right image.

We consider the influence of compact sources within the SNR by employing similar methods to Tian \& Leahy (2005), although the compact sources within G127.1+0.5 are not bright compared with those for another nearby SNR G126.2+1.6 (Tian \& Leahy 2006a).

\section{Results}

\subsection{Continuum emission from $\mathrm{G} 127.1+0.5$}

The CGPS continuum images at $408 \mathrm{MHz}$ and $1420 \mathrm{MHz}$ are shown in the upper left and right panels of Fig. 1. The lower left shows the WENSS (the Westerbork North Sky Survey, beam size $54^{\prime \prime} \times 54^{\prime \prime} \operatorname{cosec}(\delta)$, Rengelink et al. 1997) map at $327 \mathrm{MHz}$ (grey scale) with contours from the $1420 \mathrm{MHz}$ map. The $2695 \mathrm{MHz}$ Effelsberg map is reproduced in the lower right for reference (Fürst et al. 1990). The Effelsberg map has a resolution of $4.3^{\prime}$ and a brightness temperature sensitivity of $50 \mathrm{mK} T_{\mathrm{B}}$.

The $408 \mathrm{MHz}$ image for G127.1+0.5 is similar to the $408 \mathrm{MHz}$ image of Joncas et al. (1989). The southern and northern shell structures are clearly seen. The radius of northern shell is about $24^{\prime}$, that of the southern is about $17^{\prime}$. The $1420 \mathrm{MHz}$ map here shows much better detail of the fine structures in G127.1+0.5 than any previous image. A bright filament structure appears in the northern shell of the SNR, and a weak multi-filamentary structure appears in the southern part of the SNR. The $408 \mathrm{MHz}$ image of G127.1+0.5 is consistent with a lower resolution version of the $1420 \mathrm{MHz}$ image, except for the intensity of the central source. Figure 1 shows that main features of G127.1+0.5 in the $327 \mathrm{MHz}$ WENSS map are consistent with the CGPS $408 \mathrm{MHz}$ and $1420 \mathrm{MHz}$ maps.

\subsection{T-T plot spectral indices}

Bright compact sources affect the measured integrated flux densities for G127.1+0.5 and its measured spectral index $\alpha$. Thus we correct for the effects of compact sources. Table 1 lists properties of the 6 brightest compact sources which are detected within G127.1+0.5 at both $408 \mathrm{MHz}$ and $1420 \mathrm{MHz}$. 

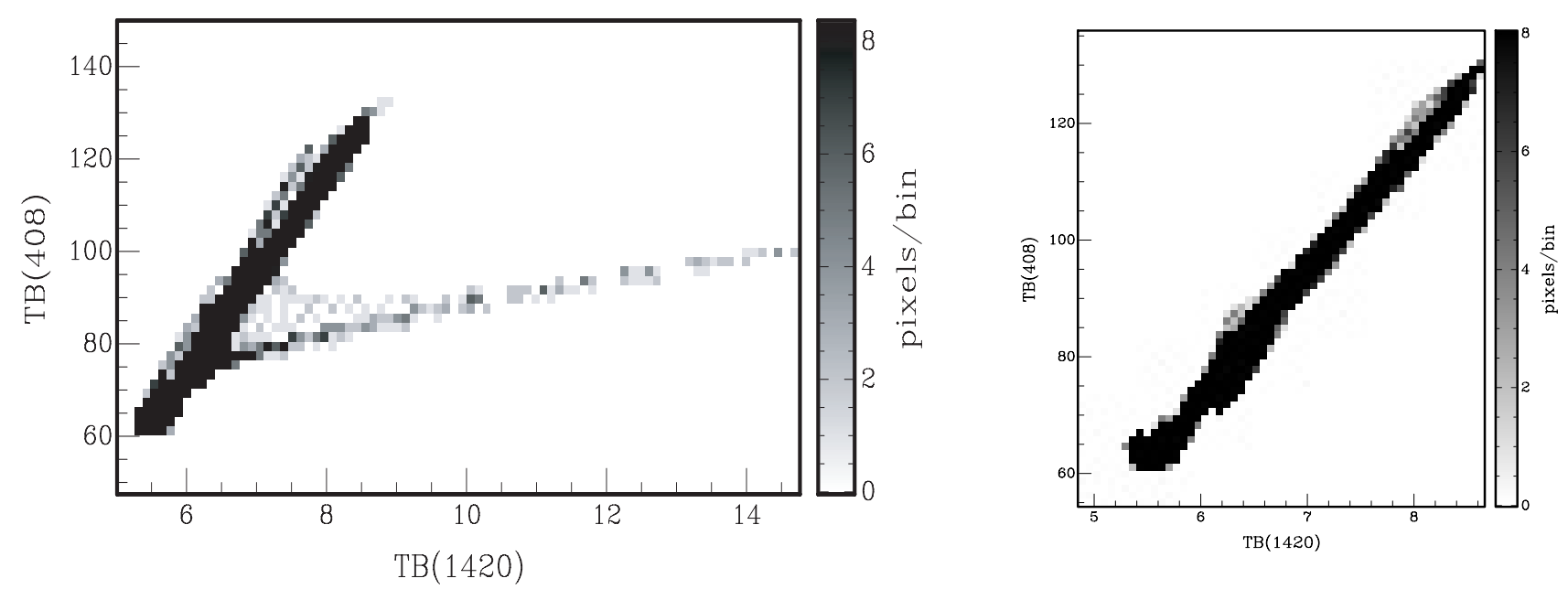

Fig. 2. Whole SNR 408-1420 MHz T-T plots. The left plot includes compact sources $(\alpha=0.47 \pm 0.01)$; the right plot has compact sources removed from the analysis $(\alpha=0.46 \pm 0.01)$.

Table 1. List of the 6 brightest compact sources and their integrated flux densities.

\begin{tabular}{cccccc}
\hline \hline $\begin{array}{c}\text { No. } \\
\end{array}$ & $\begin{array}{c}\text { GLON } \\
\text { deg }\end{array}$ & $\begin{array}{c}\text { GLAT } \\
\text { deg }\end{array}$ & $\begin{array}{c}S_{408 \mathrm{MHz}} \\
\text { mJy }\end{array}$ & $\begin{array}{c}S_{1420 \mathrm{MHz}} \\
\text { mJy }\end{array}$ & $\begin{array}{c}\text { Sp. index } \\
\alpha\end{array}$ \\
\hline 1 & 126.840 & 0.980 & $356 \pm 56$ & $187 \pm 34$ & $0.52(0.25$ to 0.79$)$ \\
2 & 127.451 & 0.445 & $264 \pm 13$ & $91 \pm 4$ & $0.86(0.81$ to 0.91$)$ \\
$3^{a}$ & 127.096 & 0.464 & $164 \pm 12$ & $62 \pm 3$ & $0.78(0.71$ to 0.85$)$ \\
$4^{b}$ & 127.110 & 0.538 & $123 \pm 11$ & $448 \pm 14$ & $-1.04(-1.11$ to -0.96$)$ \\
5 & 127.272 & 0.529 & $39 \pm 4$ & $7 \pm 1$ & $1.42(1.26$ to 1.62$)$ \\
6 & 127.585 & 0.972 & $32 \pm 3$ & $10 \pm 1$ & $0.94(0.84$ to 1.06$)$ \\
\hline
\end{tabular}

${ }^{a}$ Named $0124+627$ previously; ${ }^{b}$ named $0125+628$ previously.

First we discuss spectral indices between $408 \mathrm{MHz}$ and $1420 \mathrm{MHz}$ based on the T-T plot method. The principle of the $\mathrm{T}-\mathrm{T}$ plot method is that spectral indices $\left(T_{v}=T_{0} v^{-\beta}\right)$ are calculated from a fit of a linear relation to the $T_{1}-T_{2}$ values of all pixels within a given map region. $T_{1}$ is the brightness temperature of a map pixel at one frequency and $T_{2}$ is for the second frequency. The higher resolution image has been smoothed to the lower resolution for the T-T plot comparison. The brightness temperature spectral index $\beta$ is derived from the slope of the line. The error in spectral index is derived from the uncertainly in slope of the line. The flux density spectral index $\alpha$ $\left(S_{v} \propto v^{-\alpha}\right)$ is related to $\beta$ by $\beta=\alpha+2$. Spectral index refers to flux density spectral index $\alpha$ in this paper unless specifically noted otherwise.

For the T-T plot analysis, first a single region for the whole SNR is used, as shown in Fig. 1. This region yields the T-T plots shown in Fig. 2. Two cases are considered: using all pixels including compact sources; and excluding compact sources. Previous similar studies (Tian \& Leahy 2005, 2006a,b; Leahy $\&$ Tian 2005) have shown the second case produces better results compared to subtracting compact sources. The compact sources are usually bright compared to the SNR emission. Since the compact sources have generally a steeper spectrum than the SNR, they are seen in the T-T plot as steeper lines of points. But in case of $\mathrm{G} 127.1+0.5,0125+628$ has a spectrum between $408 \mathrm{MHz}$ and $1420 \mathrm{MHz}$ which is much flatter than the SNR's. This shows up in the left panel of Fig. 2 as a flatter line of points. Removing regions of pixels including compact sources from the analysis produces the right plot of Fig. 2.

Next, three smaller areas (labeled $A$ to $C$ in the upper right panel of Fig. 1) are selected to search for possible spatial variations in spectral index. Table 3 lists the results for the two cases of analysis: including compact sources and removing compact sources. There are no significant compact sources removed from either southern and northern shell regions (areas A and B). The influence of compact sources' flux density on the spectral index calculation is strong for the central area B. However, since the diffuse SNR emission doesn't have much brightness variation the uncertainty in the T-T plot derived spectral index is large. From now on we discuss spectral indices derived with compact sources removed, unless specified otherwise. There is no evidence at $1 \sigma$ for spatial variations in spectral index within G127.1+0.5.

\subsection{Central compact sources within the SNR}

Before discussing G127.1+0.5, we consider the central sources since the flux densities of the SNR must be corrected for the flux densities of all compact sources including the central sources. Flux densities for the two central compact sources $0124+627$ and $0125+628$ were determined from the $408 \mathrm{MHz}$ and $1420 \mathrm{MHz}$ maps. $0124+627$ is a steep spectrum compact source with spectral index of 0.78 between $408 \mathrm{MHz}$ and $1420 \mathrm{MHz}$. We use this spectral index and its flux density at $1420 \mathrm{MHz}$ to estimate its flux density in other frequencies. $0125+628$ 's low-frequency turnover radio spectrum is confirmed based on data at six frequencies (Table 2, Fig. 3). The current analysis is improved comparing with previous work (Joncas et al. 1989; Salter et al. 1978), since we have subtracted the contributions from $0124+627$ to $0125+628$ 's flux densities, which were included in the previous studies.

Different model spectra for $0125+628$ are considered here (e.g. Longair 1981; Leahy \& Roger 1998). If the spectrum has a turnover due to electron energy losses, it could be due to synchrotron or inverse Compton losses at high energy or due to 
Table 2. Integrated flux densities of the compact source $0125+628$.

\begin{tabular}{cccccc}
\hline \hline $\begin{array}{c}S_{408 \mathrm{MHz}} \\
\mathrm{mJy}\end{array}$ & $\begin{array}{c}S_{608 \mathrm{MHz}} \\
\mathrm{mJy}\end{array}$ & $\begin{array}{c}S_{1420 \mathrm{MHz}} \\
\mathrm{mJy}\end{array}$ & $\begin{array}{c}S_{2695 \mathrm{MHz}} \\
\mathrm{mJy}\end{array}$ & $\begin{array}{c}S_{5.0 \mathrm{GHz}} \\
\mathrm{mJy}\end{array}$ & $\begin{array}{c}S_{8.7 \mathrm{GHz}} \\
\mathrm{mJy}\end{array}$ \\
\hline $123 \pm 11^{a}$ & $210 \pm 8^{b}$ & $448 \pm 14^{a}$ & $508 \pm 35^{c}\left(38^{*}\right)$ & $480 \pm 30^{c}\left(23^{*}\right)$ & $482 \pm 30^{c}\left(15^{*}\right)$ \\
\hline
\end{tabular}

* Flux density from nearby compact source $0124+627$ which is included in present flux density of 0125-628 (see text). ${ }^{a}$ This paper; ${ }^{b}$ Pauls et al. (1982); ${ }^{c}$ Salter et al. (1978).

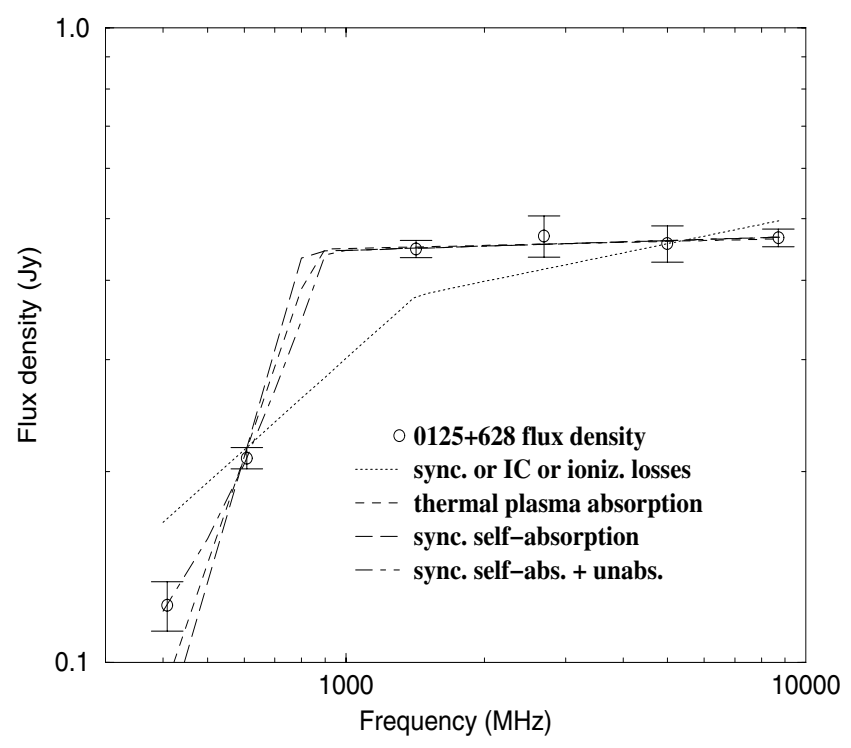

Fig. 3. Radio spectrum of $0125+628$ and model fits (see text).

ionization losses at low energy. In the former case the electron power-law injection spectral index, $\gamma$, is related to the low frequency radio spectral index, $\alpha_{\text {low }}$ by $\alpha_{\text {low }}=(\gamma-1) / 2$ and the radio spectral index at high frequency is increased by 0.5 . In the latter case, the electron power-law injection spectral index, $\gamma$, is related to the high frequency radio spectral index, $\alpha_{\text {hi }}$ by $\alpha_{\text {hi }}=(\gamma-1) / 2$ and the radio spectral index at low frequency is decreased by 0.5 . The best fit model for both cases is shown in Fig. 3. It has $\alpha_{\text {low }}=-0.65, \alpha_{\text {hi }}=-0.15$ with break frequency $1400 \mathrm{MHz}$ and $\chi^{2}=49$. It is inconsistent with the data as it doesn't give a sharp enough turnover. Absorption mechanisms give a sharper turnover. Absorption by a dense thermal plasma in front of the radio source gives an exponential cutoff in frequency which is too sharp, but one can have absorption by a thermal plasma intermixed with the radiating electrons, which gives a power-law cutoff where the low frequency radio spectral index is decreased by 2.1 with respect to the high frequency index. This model fits the data with $\alpha_{\text {low }}=-2.26, \alpha_{\text {hi }}=-0.16$ with break frequency $860 \mathrm{MHz}$ and $\chi^{2}=8.3$. Synchrotron selfabsorption gives a low frequency spectral index of -2.5 , and also fits the data with best fit parameters: $\alpha_{\mathrm{hi}}=-0.02$ with break frequency $810 \mathrm{MHz}$ and $\chi^{2}=16.2$. A better fitting model is one for a source with synchrotron self-absorption but with some part of the source not subject to self-absorption. This can easily occur if the source has regions with high and low density of synchrotron emitting electrons. This model fits the data very well $\left(\chi^{2}=0.2\right)$ with $\alpha_{\mathrm{hi}}=-0.02$ with break frequency $900 \mathrm{MHz}$ for the high density region, and relative contribution of the low density region (with $\alpha$ fixed equal to $\alpha_{\mathrm{hi}}$ ) of $20 \%$. In summary, a simple model with synchrotron self-absorption fits the spectrum of $0125+628$. This model is used to calculate the contribution of $0125+628$ to the integrated flux density of G127.1+0.5 at frequencies where $0125+68$ has not been directly measured.

\subsection{Integrated flux densities and spectral indices}

Integrated flux densities of G127.1+0.5 with diffuse background subtracted were derived at $408 \mathrm{MHz}$ and $1420 \mathrm{MHz}$. The resulting $408 \mathrm{MHz}$ to $1420 \mathrm{MHz}$ spectral index, using flux densities without compact sources, is $0.43 \pm 0.10$. Table 4 lists the flux densities at 408 and $1420 \mathrm{MHz}$ and spectral indices with and without the compact sources within G127.1+0.5. Compact sources contribute about $6 \%$ at $408 \mathrm{MHz}$ and $8 \%$ at $1420 \mathrm{MHz}$, and generally have no significant effect on spectral index except for area B. The whole SNR spectral index derived from integrated flux densities is consistent with the whole SNR spectral index $(0.46 \pm 0.01)$ derived by the T-T plot method.

Published integrated flux densities and errors for the SNR at other frequencies are given in Table 5. For 408 and $1420 \mathrm{MHz}$ the values in Table 5 already have compact source flux density removed. Fürst et al. (1984) gave a flux density based on the $2965 \mathrm{MHz}$ Effelsberg image with resolution $4.4 \times 4.4 \mathrm{arcmin}$, but we obtain a new value from the Effelsberg $2695 \mathrm{MHz}$ image with a little higher resolution $4.3 \times 4.3$ arcmin $8.3 \pm 0.8 \mathrm{Jy}$ and use this instead. We fit the resulting flux density values with a power-law to obtain the spectral index. Figure 4 shows the corrected flux densities and the best-fit power-law with $\alpha=$ $0.49,90 \%$ uncertainty range 0.42 to 0.56 .

\subsection{HI emission}

We have searched the CGPS HI images for features in the HI which might relate to the morphology of G127.1+0.5. There is emission along the edge of northern and southern shells of $\mathrm{G} 127.1+0.5$ in the velocity range -12 to $-16 \mathrm{~km} \mathrm{~s}^{-1}$, and only in this range. Figure 5 shows maps of $\mathrm{HI}$ emission in the six velocity channels in this range. One contour of $1420 \mathrm{MHz}$ continuum emission is shown to indicate the outline of G127.1+0.5. The emission along the edge of G127.1+0.5 can alternately be described as a depression in the HI emission. This HI association with $\mathrm{G} 127.1+0.5$ is similar to other accepted HI associations with supernova remnants and with similar HI velocity ranges $\left(\sim 10-20 \mathrm{~km} \mathrm{~s}^{-1}\right)$, e.g. for the Cygnus Loop (Leahy 2003) and DA530 (Landecker et al. 1999). 
Table 3. 408-1420 MHz T-T plot spectral indices with and without Compact Sources (CS).

\begin{tabular}{ccc}
\hline \hline Area & $\begin{array}{c}\alpha \\
\text { including CS }\end{array}$ & $\begin{array}{c}\alpha \\
\text { CS removed }\end{array}$ \\
& $0.49 \pm 0.01$ & $0.49 \pm 0.01$ \\
A & $0.50 \pm 0.17$ & $0.7 \pm 0.3$ (manual fit) \\
B & $0.46 \pm 0.06$ & $0.46 \pm 0.06$ \\
\hline C & $0.47 \pm 0.01$ & $0.46 \pm 0.01$ \\
\hline All G127.1+0.5
\end{tabular}

Table 4. Integrated flux densities and spectral indices of G127.1+0.5 with and without compact sources (CS).

\begin{tabular}{cccc}
\hline \hline Source & $S_{408 \mathrm{MHz}}$ & $S_{1420 \mathrm{MHz}}$ & $\alpha$ \\
& $\mathrm{Jy}$ & $\mathrm{Jy}$ & \\
\hline G127.1+0.5 & $17.1 \pm 1.7$ & $10.0 \pm 0.8$ & $0.43 \pm 0.10$ \\
$\mathrm{G} 127.1+0.5 \& \mathrm{CS}$ & $18.1 \pm 1.8$ & $10.8 \pm 0.9$ & $0.41 \pm 0.10$ \\
\hline
\end{tabular}

Table 5. Integrated Flux Densities (FD) of G127.1+0.5.

\begin{tabular}{cccc}
\hline \hline $\begin{array}{c}\text { Freq. } \\
\mathrm{MHz}\end{array}$ & $\begin{array}{c}H P B W \\
\text { arcmin }\end{array}$ & $\begin{array}{c}\text { FD } \\
\mathrm{Jy}\end{array}$ & References for FD \\
\hline 178 & $23 \times 19$ & $37.0 \pm 7.0$ & Salter et al. (1978) \\
408 & $3.5 \times 3.9$ & $17.9 \pm 2.0^{*}$ & Joncas et al. (1989) \\
408 & $3.4 \times 3.8$ & $17.1 \pm 1.7^{*}$ & this paper \\
865 & $14.5 \times 14.5$ & $14.9 \pm 0.8$ & Reich et al. (2003) \\
1420 & $1 \times 1.1$ & $10.1 \pm 0.8^{*}$ & Joncas et al. (1989) \\
1420 & $1 \times 1.1$ & $10.0 \pm 0.8^{*}$ & this paper \\
2695 & $4.4 \times 4.4$ & $8.4 \pm 0.6$ & Fürst et al. (1984) \\
2695 & $4.3 \times 4.3$ & $8.3 \pm 0.8$ & see text \\
4850 & $2.6 \times 2.6$ & $6.2 \pm 0.4$ & Fürst et al. (1984) \\
\hline
\end{tabular}

* Means CS within the SNR have been subtracted.

\section{Discussion}

\subsection{The distance and age of $\mathrm{G} 127.1+0.5$}

There are no previous reliable distance estimates for G127.1+0.5. Joncas et al. (1989) suggested a range of $2-5 \mathrm{kpc}$. Previous observations didn't provide any compelling evidence to associate G127.1+0.5 with either the compact sources within its area (Spinrad et al. 1979; Pauls et al. 1982; Goss \& van Gorkom 1984) or with the superposed cluster NGC 559 (Pauls et al. 1982). Furist et al. (1984) inspected images from the Maryland $21 \mathrm{~cm} \mathrm{HI} \mathrm{Line} \mathrm{survey} \mathrm{(Westerhout} \mathrm{1972)} \mathrm{and} \mathrm{re-}$ ported a hole between velocities -70 to $-90 \mathrm{~km} \mathrm{~s}^{-1}$ centered on G127.1+0.5. Our high resolution $\mathrm{HI}$ images do not confirm this hole: only in velocity range -12 to $-16 \mathrm{~km} \mathrm{~s}^{-1}$, is there emission coincident with G127.1+0.5 (Fig. 5).

The HI radial velocity for G127.1 +0.5 of $-14 \mathrm{~km} \mathrm{~s}^{-1}$ gives a distance estimate based on the galactic rotation curve. We take $R_{0}=8.5 \mathrm{kpc}, V_{0}=220 \mathrm{~km} \mathrm{~s}^{-1}$. If G127.1+0.5 is within $\sim 2 \mathrm{kpc}$, then the circular velocity at the SNR is also $220 \mathrm{~km} \mathrm{~s}^{-1}$, which gives a distance of $1.15 \mathrm{kpc}$. If we make the extreme assumption that G127.1+0.5 is far away and that the circular velocity at the SNR is $250 \mathrm{~km} \mathrm{~s}^{-1}$ we find an upper limit to the distance of $2.9 \mathrm{kpc}$. The open cluster NGC 559 located on the face of the SNR has a distance 0.9-1.3 kpc (Blair et al. 1980; Xilouris et al. 1993), consistent with our estimate

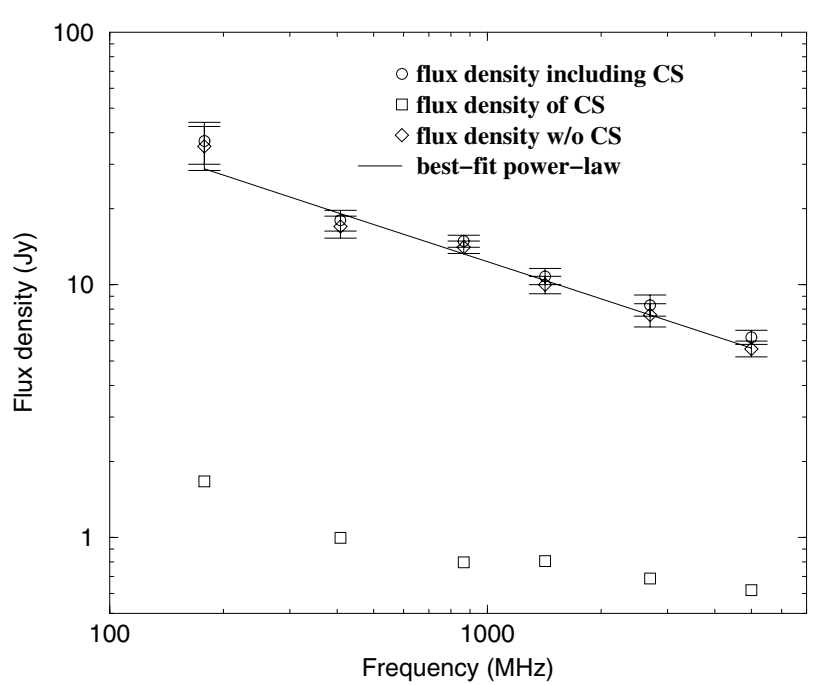

Fig. 4. Radio spectrum of G127.1+0.5. The best-fit spectral index is 0.49 , with $\chi^{2}=3.9$, for a fit to 6 frequencies in range $178-4850 \mathrm{MHz}$.

of G127.1+0.5's distance. The association of NGC 559 with G127.1+0.5 is also supported by detection of optical emission in the area of G127.1+0.5 (Xilouris et al. 1993) which generally indicates a close distance to us.

At $1.15 \mathrm{kpc}$ distance, G127.1+0.5 has a mean radius of $r=8 \mathrm{pc}$. Summing the HI channel maps from -12 to $16 \mathrm{~km} \mathrm{~s}^{-1}$, taking the difference between the rim and center of G127.1+0.5, and converting to column density, we find an excess of $\simeq 10^{20} \mathrm{~cm}^{-2}$ along the rim. This yields an estimate of the local density $n_{0}=10^{20} \mathrm{~cm}^{-2} /(2 r) \simeq 2 \mathrm{~cm}^{-3}$. Applying a Sedov model (e.g. Cox 1972), for a typical explosion energy of $E=0.5 \times 10^{51} \mathrm{erg}\left(\epsilon_{0}=E /\left(0.75 \times 10^{51} \mathrm{erg}\right)=2 / 3\right)$, yields an age of $\simeq 3 \times 10^{4}$ yr. However, the Sedov age is large enough that G127.1+0.5 should have entered the dense shell phase. For $n_{0}=2 \mathrm{~cm}^{-3}$ the shock radius and the age when time the SNR enters the dense shell phase are $15.8 \mathrm{pc}$ and $2.3 \times 10^{4} \mathrm{yr}$, so G127.1+0.5 should be just past entering the dense shell phase. The Sedov estimate for shocked gas temperature just interior to the shell at the time of the start of the dense shell phase is still valid: $7 \times 10^{5} \mathrm{~K}$. In the cooling phase, the material which is recently shocked cools rapidly to add mass to the dense shell. The dense shell emits mostly in the ultraviolet, which will not be observable through the column density to the SNR. This is $\simeq 2 \times 10^{21} \mathrm{~cm}^{-2}$, derived from either the extinction to NGC 559 or the HI channel maps for velocities $<-14 \mathrm{~km} \mathrm{~s}^{-1}$. Similarly the low luminosity soft X-ray emission from the SNR interior would not be visible, consistent with its lack of detection in the Rosat All-Sky Survey data.

\subsection{Radio spectra of $\mathrm{G} 127.1+0.5$ and $0125+628$}

Figure 4 shows the radio spectrum of G127.1+0.5. Excluding compact source flux densities, the spectrum is consistent $\left(\chi^{2}=\right.$ 3.9) with a power-law with $\alpha=0.49,90 \%$ confidence range of 0.42 to 0.56 . The compact sources do not strongly affect the SNR's spectrum. The spectral index from integrated flux densities agrees with both the T-T plot spectral index $(0.46 \pm 0.01)$ 

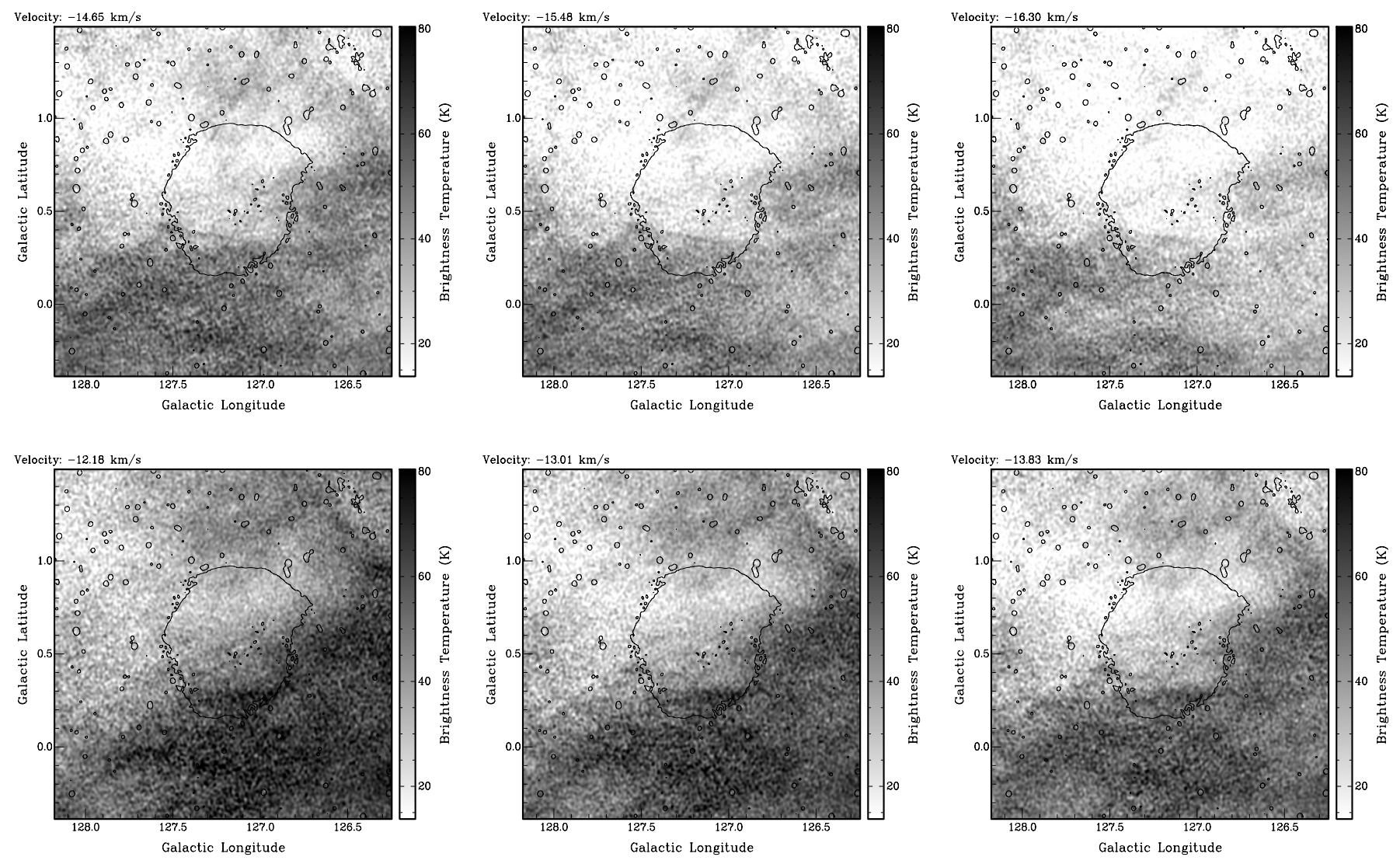

Fig. 5. $\mathrm{HI}$ emission in the field centered on G127.1+0.5 from -12 to $-16 \mathrm{~km} \mathrm{~s}^{-1}$. The radial velocity of each map is indicated at its top left corner. The outline of G127.1+0.5 in $1420 \mathrm{MHz}$ continuum emission is indicated by the contour at $6 \mathrm{~K} T_{\mathrm{B}}$.

and the 408-1420 MHz integrated flux density spectral index $(0.43 \pm 0.10)$.

The central compact sources $0125+628$ and $0124+627$ have been previously studied because they are located near the center of the SNR. Optically $0125+628$ was associated with an elliptical galaxy by Kirshner \& Chevalier (1978). Pauls et al. (1982) confirm that $0125+628$ is a background radio source due to its large column density. Kaplan et al. (2004) find a hard spectrum X-ray source associated with $0125+628$ consistent with $0125+628$ being an AGN, but no $\mathrm{X}$-ray emission from $0124+627$. Spitler \& Spangler (2005) observe $0125+628$ with the VLBA and find no excess scattering associated with G127.1+0.5.

For $0124+627$, we find no evidence of deviation from a power-law spectrum comparing our 408 and $1420 \mathrm{MHz}$ data to the $608 \mathrm{MHz}$ value of Pauls et al. (1982). For 0125+628 we obtain a significantly improved spectrum (Fig. 3) by correcting published values for confusion with $0124+627$. The strong spectral break cannot be reproduced by synchrotron, inverse Compton, or ionization loss models. Synchrotron selfabsorption or thermal plasma absorption (if mixed with the emitting plasma) can give the observed break, with the thermal plasma absorption model somewhat better fitting due to its smaller spectral break. However since the synchrotron selfabsorption takes place only in high density emitting regions, one might expect to have some fraction of the source at low density and free of self-absorption. This last case provides the best fit to the observed spectrum and yields an unabsorbed fraction of $\simeq 20 \%$. Since $0125+628$ is known to be an AGN, one would expect synchrotron self-absorption similar to what is seen for a number of other AGN. Thus we prefer the last model as a description of the observed spectrum.

\section{Conclusion}

We present new maps of G127.1+0.5 at $408 \mathrm{MHz}$ and 1420 $\mathrm{MHz}$ and study the radio spectrum, corrected for point source flux densities, using different methods. All results are consistent with the best determined value from the T-T plot method of $0.46 \pm 0.01$. We find no evidence for spectral index variation across G127.1+0.5. A probable association of HI features with G127.1+0.5 yields a distance of $1.15 \mathrm{kpc}$ for G127.1+0.5, consistent with association with the open cluster NGC 559. The HI deficit associated with the SNR gives a local density of $\simeq 2 \mathrm{~cm}^{-3}$, which yields a dense shell transition radius of $7.9 \mathrm{pc}$, roughly equal to the observed radius. So G127.1+0.5 should be approximately at the age where it enters the dense shell phase, and have an age of $2-3 \times 10^{4} \mathrm{yr}$.

Acknowledgements. We acknowledge support from the Natural Sciences and Engineering Research Council of Canada. The DRAO is operated as a national facility by the National Research Council of Canada. The Canadian Galactic Plane Survey is a Canadian project with international partners. 


\section{References}

Blair, W. P., Kirshner, R. P., Gull, T. R., Sawyer, D. L., \& Parker, R. A. R. 1980, ApJ, 242, 592

Cox, D. 1972, ApJ, 178, 159

Fürst, E., Reich, W., \& Steube, R. 1984, A\&A, 133, 11

Fürst, E., Reich, W., Reich, P., \& Reif, K. 1990, A\&AS, 85, 691

Goss, W. M., \& van Gorkom, J. H. 1984, A\&A, 5, 425

Haslam, C. G. T., Salter, C. J., Stoffel, H., \& Wilson, W. W. 1982, A\&AS, 47, 1

Higgs, L. A., \& Tapping, K. F. 2000, AJ, 120, 2471

Joncas, G., Roger, R. S., \& Dewdney, P. E. 1989, A\&A, 219, 303

Kaplan, D. L., Frail, D. A., Gaensler, B. M., et al. 2004, ApJS, 153, 269

Kirshner, R. P., \& Chevalier, R. A. 1978, Nature, 276, 480

Landecker, T. L., Routledge, D., Reynolds, S. P., et al. 1999, ApJ, 527, 866

Longair, M. S. 1981, High Energy Astrophysics (Cambridge University Press)

Leahy, D. A. 2003, AJ, 586, 224

Leahy, D. A., \& Roger, R. S. 1998, ApJ, 505, 784

Leahy, D. A., \& Tian, W. W. 2005, A\&A, 440, 929
Milne, D. K. 1988 in Supernova Remnants and the Interstellar Medium, ed. R. S. Roger, \& T. L. Landecker (Cambridge: University Press), 351

Pauls, T. 1977, A\&A, 59, L13

Pauls, T., van Gorkom, J. H., Goss, W. M., et al. 1982, A\&A, 112, 120

Reich, W., Reich, P., \& Fürst, E. 1990, A\&AS, 83, 539

Reich, W., Reich, P., \& Fürst, E. 1997, A\&AS, 126, 413

Reich, W., Zhang, X., \& Fürst, E. 2003, A\&A, 408, 961

Rengelink, R. B., Tang, Y., de Bruyn, A. G., et al. 1997, A\&AS, 124, 259

Salter, C. J., Pauls, T., \& Haslam, C. G. T. 1978, A\&A, 66, 77

Spitler, L. G., \& Spangler, S. R. 2005, ApJ, 632, 932

Spinrad, H., Stauffer, J., \& Harlan, E. 1979, PASP, 91, 619

Taylor, A. R., Gibson, S. J., Peracaula, M., et al. 2003, AJ, 125, 3145

Tian, W. W., \& Leahy, D. A. 2005, A\&A, 436, 187

Tian, W. W., \& Leahy, D. A., 2006a, A\&A, 447, 205

Tian, W. W., \& Leahy, D. A. 2006b, ChJAA, accepted [arXiv: astro-ph/0511320]

Westerhout, G. 1972, Maryland-Greenbank Galactic $21 \mathrm{~cm}$ Survey

Xilouris, K. M., Papamastorakis, J., Paleologou, E. V., Andredakis, Y., \& Haerendel, G. 1993, A\&A, 270, 393 